\title{
Application of single-cell RNA sequencing technology in liver diseases: a narrative review
}

\author{
Lian $\mathrm{He}^{1}$, Anjing Lu ${ }^{1,2}$, Lin Qin ${ }^{1}$, Qianru Zhang ${ }^{1}$, Hua Ling ${ }^{3}$, Daopeng Tan ${ }^{1}$, Yuqi He ${ }^{1}$ \\ ${ }^{1}$ The Key Laboratory of Basic Pharmacology of Minstry of Education and Joint International Research Laboratory of Ethnomedicine of Ministry \\ of Education, School of Pharmacy, Zunyi Medical University, Zunyi, China; ${ }^{2}$ Shanghai Nature-Standard Technology Service Co., Ltd., Shanghai, \\ China; ${ }^{3}$ School of Pharmacy, Georgia Campus-Philadelphia College of Osteopathic Medicine, Suwanee, GA, USA \\ Contributions: (I) Conception and design: Y He; (II) Administrative support: Q Zhang; (III) Provision of study materials or patients: L He; (IV) \\ Collection and assembly of data: L He, A Lu; (V) Data analysis and interpretation: L He; (VI) Manuscript writing: All authors. (VII) Final approval \\ of manuscript: All authors. \\ Correspondence to: Yuqi He; Daopeng Tan. School of Pharmacy, Zunyi Medical University, Zunyi 563000, China. \\ Email: HyqJeff@foxmail.com; 1474755772@qq.com.
}

Objective: This review aimed to summarize the application of single-cell transcriptome sequencing technology in liver diseases.

Background: The increasing application of single-cell ribonucleic acid (RNA) sequencing (scRNA-seq) in life science and biomedical research has greatly improved our understanding of cellular heterogeneity in immunology, oncology, and developmental biology. scRNA-seq has proven to be a powerful tool for identifying and classifying cell subsets, characterizing rare or small cell subsets and tracking cell differentiation along the dynamic cell stages. Globally, liver disease has high rates of morbidity and mortality, and its exact pathological mechanism remains unclear, current treatment options are limited to clearance of the underlying cause or liver transplantation, which cannot overwhelm and cure liver diseases. scRNA-seq provides many novel insights for healthy and diseased livers.

Methods: In this review, we searched for related articles in the PubMed database and summarized the advances of scRNA-seq in revealing the molecular mechanisms of liver development, regeneration, and disease. We also discussed the challenges and future application potential of scRNA-seq, which is expected to enhance the ability to explore the field of liver research and accelerate the clinical application of liver precision medicine.

Conclusions: With the continuous improvement of scRNA-seq technology, scRNA-seq is expected to unlock new avenues for liver biology exploration, liver disease diagnosis, and personalized treatment, which will pave the way for breakthrough innovation in personalized medicine.

Keywords: Single cell RNA sequence (scRNA-seq); cellular heterogeneity; liver disease; precision medicine

Submitted Aug 17, 2021. Accepted for publication Oct 14, 2021.

doi: $10.21037 / \mathrm{atm}-21-4824$

View this article at: https://dx.doi.org/10.21037/atm-21-4824

\section{Introduction}

As the largest solid organ in the body, the liver contains heterogeneous cell types with unique spatial, molecular, and functional characteristics (1). Most hepatocytes form liver parenchymal cells (2), while non-parenchymal cells (NPCs) include liver sinusoidal endothelial cells (LSECs), Kupffer cells (KCs), and hepatic stellate cells (HSCs), bile duct cells, and other immune cell populations mainly composed of natural killer T cells $(3,4)$. Globally, liver disease has high rates of morbidity and mortality $(5,6)$. Studies have shown that liver disease persists through the coordination of liver parenchymal cells and NPCs (7-10). The pathological mechanisms of liver disease may involve a large and complex network of cellular and molecular mechanisms, but there is 
a lack of detailed analysis defining the cellular and molecular mechanisms that drive these diseases, and current treatment options are limited to clearance of the underlying cause or liver transplantation. Therefore, uncovering the molecular mechanism of hepatocyte heterogeneity and studying new effective therapeutic strategies remains challenging.

Cells are the basic structural and functional units of organisms, and the potential heterogeneity within cells is a basic characteristic of cellular homeostasis and development (11). Traditional gene expression analysis techniques are limited to the cell population level and can only obtain the average genetic information reflecting the dominant cell subpopulation, while the role of the secondary cell subpopulation is often ignored (12). Thus, the biological behavior of individual cells may also be ignored, resulting in the loss of cell heterogeneity information.

The emerging scRNA-seq technique possesses advantages including the ability to analyze transcriptome heterogeneity that is masked in bulk RNA, as well as the ability to identify rare cell types and new cell states. This has profound implications for understanding the physiological functions and biological processes associated with human disease $(13,14)$. In the era of immunotherapy and precision medicine, higher resolution sequencing data is needed to characterize heterogeneous tissues and complex diseases, such as chronic liver disease. Recently, studies using scRNA-seq have revealed the emergence of diverse liver parenchymal and NPCs from their endodermal and mesodermal ancestors during embryonic development, found the zone-specific alterations of LSECs in Cirrhosis $(15,16)$, identified subsets of HSCs and provided a transcriptional roadmap for the activation of HSCs during liver fibrosis $(17,18)$. ScRNA-seq not only explored the heterogeneity of liver-resident immune cells but also revealed the presence of triggering receptor expressed on myeloid cells 2(TREM2)+ macrophages, ACKR1+ and PLVAP+ endothelial cells expanding in cirrhosis in fibrotic livers (19-21).

Through scRNA-seq, cell transcription maps can be mapped with unprecedented resolution, and cellular heterogeneity in liver development and disease can be described (22). This will greatly promote the understanding of human liver health and disease.

In this paper, we discussed the scRNA-seq technique, data visualization analysis methods and how it has changed our understanding of liver biology and disease pathogenesis, and unlocked new strategies for finding effective novel treatments for patients with liver diseases. We also predicted the technical improvements and clinical applications of scRNA-seq in the future as it is still limited by cost, technology, complexity of data processing, etc. We present the following article in accordance with the Narrative Review reporting checklist (available at https:// dx.doi.org/10.21037/atm-21-4824).

\section{Methods}

In the current narrative review, we conducted a literature search for papers published up to June 2021 on scRNA-seq and liver in the PubMed database, all of the included studies were written in English. The vast majority of researched literature was focused on the application of single-cell RNA sequencing technology in liver.

\section{Single-cell transcriptome sequencing technology}

\section{Workflow of single-cell transcriptome sequencing}

With the innovation of sequencing technology, numerous scRNA-seq technologies have been developed, which have different methods of cell capture and amplification, messenger ribonucleic acid (mRNA) transcript length, number of captured target cells, and reading depth of each cell (23). Each approach has its own unique advantages and disadvantages, but overall, all of the scRNA-seq techniques developed to date follow a common workflow: single-cell isolation and library generation, sequencing, and visual analysis.

\section{Single-cell isolation and library generation}

Single-cell isolation is the limiting step of scRNA-seq, and library preparation is also a key factor that improves the throughput of high-throughput scRNA-seq research. In 2009, Tang et al. (24) detected and captured only six cells through micromanipulation. In recent years, commercial instruments developed by $10 \times$ Genomics can simultaneously prepare approximately 48,000 single cells from eight different samples (25). It is clear that scRNA-seq technology has been rapidly developed. Once successfully captured, individual cells are reverse transcribed into complementary deoxyribonucleic acid (cDNA), which is then amplified by polymerase chain reaction (PCR) or in vitro transcription (IVT) to generate high-throughput sequencing libraries (26).

\section{ScRNA-seq platform}

Methods for isolating individual cells for RNA sequencing 
vary by the number of cells isolated (high or low throughput) and the way in which cells are selected (biased or unbiased) (27). The available platforms fall into two categories according to the downstream methods: dropletbased [e.g., Drop-seq (28), inDrop (29), 10× Chromium Genomics (25), and Seq-well (30)] and plate-based [e.g., STRT-seq (31), SmartSeq (32), SmartSeq2 (33), and MARS-Seq (34)]. Droplet-based approaches are suitable for generating large numbers of cells to identify subsets of cells in a complex tissue or tumor sample such as the liver. The three most widely used platforms are Dropseq, inDrop, and $10 \times$ Chromium Genomics, each of which uses microfluidics. Individual cells are labeled with individual magnetic beads with unique barcodes, and each mRNA transcript is also associated with a unique molecular identifier (UMI). Methods for generating a unit sequencing database are based on the full-length and tag-based methods $(35,36)$. Compared with tag-based methods, the fulllength scRNA-seq method has incomparable advantages in isomer use analysis, allele expression detection, as well as RNA editing and identification due to its advantages in transcription coverage (32). In addition, some scRNA-seq techniques, such as SUPeR-seq (37) and MATQ-seq (38), can simultaneously capture polyA+ and polyA-RNA, which has profound implications for the sequencing of long noncoding RNAs (lncRNAs) and circular RNAs (circRNAs).

A few issues need to be considered in order to decide which one suits any particular researcher's needs best. Plate-based and droplet platforms are suitable for normal and small cells, while full-length methods can also be used independently of all cell types or sizes, allowing analysis of large and rod-shaped cells, such as adult cardiomyocytes (39). Plate based protocols can provide experimental flexibility for long-term stored cell samples. Researchers interested in the details of each cell should choose highly sensitive protocols, such as SMART-seq2, MARS-Seq, which are more suitable for fewer cells $(35,40,41)$. Droplet-based techniques are often used as high-throughput studies, which are suitable for generating large numbers of cells to identify subpopulations of cells in complex tissues or tumour samples like the liver. Full-length methods are superior for isoform analysis, allele detection and RNA editing identification (32).

Currently, scRNA-seq includes a variety of technologies, and the selection of sequencing platforms should be guided by biological questions, with the appropriate technology or combination of technologies selected based on the context of the study design and the desired endpoint.

\section{scRNA-seq data processing and visual analysis}

Due to the relative lack of starting materials, improved resolution, loss of cDNA synthesis, and error of cDNA amplification, the scRNA-seq data set has considerable technical noise and serious quantitative error (42). Therefore, after the completion of sequencing, quality control of the data should be carried out to assess the gene expression level of qualified cells (43) and the gene expression level of qualified cells can be evaluated by FASTQC, Qualimap2, RSeQ and CQoRT (43-46), tools such as HTSeq and WemIQ $(47,48)$ can align reads to a reference genome and calculate the relative quantization of mRNA molecules for each gene in each cell, reads can be assigned to reference annotated genes via STAR (49) and feature Counts (50). Subsequently, public platforms (such as Cell Ranger of $10 \times$ Genomics) should be used to generate the feature barcode matrix and create the original gene expression count matrix (43). Due to factors such as reverse transcription efficiency, primer capture efficiency, and errors related to UMIs, the proportion of RNA captured by each cell may be different $(51,52)$, and the difference in the total amount of UMIs or reads in each cell may be caused by technical factors rather than biological variation. Therefore, normalization is critical to reveal the true biological heterogeneity of the dataset, and the use of specialized normalization methods, such as SCnorm and sctransform, can unlock heterogeneity in the dataset (52). Finally, numerous toolkits are used for visualization and data analysis. Table 1 summaries some visualization analysis toolkits that are representative in the scRNA-seq field (53-62).

\section{Biology of the liver at the single-cell level}

\section{Hepatocyte mapping and liver partitioning}

MacParland et al. (63) mapped the transcription profiles of 8,444 human hepatocytes and NPCs at singlecell resolution and identified 20 hepatocyte subsets. Subsequently, Ramachandran et al. (19) used the healthy cell bank to generate a single cell atlas of human liver NPCs and identified 21 cell subsets. Zhao et al. (21) given a global glimpse on B cell and plasma cell subsets and revealed the heterogeneity and functional diversity of liver-resident immune cells in human. Aizarani et al. (64) revealed a human liver cell atlas and analyzed the heterogeneity of LSECs, KCs, and hepatocyte subtypes, as well as the transcriptomic range of hepatocytes. These results suggest that the heterogeneity of the epithelial cell 
Table 1 scRNA-seq visualisation analysis toolkits

\begin{tabular}{lll}
\hline Toolkits & Synopsis & Reference \\
\hline Seurat & $\begin{array}{l}\text { Quality control, cell clustering, analysis of differentially } \\
\text { expressed genes, identification of marker genes }\end{array}$ & $\begin{array}{l}\text { Butler et al. Nat Biotechnol 2018 (53) } \\
\text { Satija et al. Nat Biotechnol 2015 (54) }\end{array}$ \\
$\begin{array}{l}\text { Monocle } \\
\text { Velocyto }\end{array}$ & Pseudo-time analysis & Trapnell et al. Nat Biotechnol 2014 (55) \\
StemID & Lineage tracing & Grün et al. Cell Stem Cell 2016 (57) \\
SCODE & Analysis single-cell gene regulatory network & Matsumoto et al. Bioinformatics 2017 (58) \\
SCENIC & & Van de Sande et al. Nat Protoc 2020 (59) \\
CellPhoneDB & Infer cell-cell communication from combined & Efremova et al. Nat Protoc 2020 (60) \\
NicheNet & expression of multi-subunit ligand-receptor complexes & Browaeys et al. Nat Methods 2020 (61) \\
SingleR & Automatic cluster annotation & Aran et al. Nat Immunol 2019 (62) \\
\hline
\end{tabular}

scRNA, single cell RNA sequence.

adhesion molecule (EPCAM)+ cell population and the new EPCAM+ trophoblast cell-surface antigen 2 (TROP2) population may be involved in homeostasis transition, liver regeneration, chronic disease occurrence, and tumor formation. Mapping the human liver in health and disease with single-cell resolution will facilitate the construction of human liver models, which is essential for understanding the pathogenesis and treatment of liver disease.

Liver zonation refers to the differential expression of liver genes along the lobe axis of hepatic lobules, which is a prominent aspect of hepatocyte heterogeneity (65). Studies based on normal human livers have shown that the liver is divided into nine levels that have different gene expression profiles, and hepatocytes are divided into pericentral hepatocytes, periportal hepatocytes, and middle hepatocytes $(63,66,67)$. Pathway analysis has demonstrated that periportal hepatocytes enrich oxidation and fatty acid metabolism genes, while middle hepatocytes enrich cytochrome P450 xenometabolic genes (64). Dobie et al. (68) found through scRNA-seq of healthy and fibrotic mouse hepatocytes that HSCs could be divided into two regions: portal vena-associated hepatic stellate cells (PaHSCs) and central vena-associated hepatic stellate cells (CaHSCs). Halpern et al. (67) found that $50 \%$ of hepatocyte genes were significantly zoned as detected by scRNA-seq combined with single-molecule fluorescence in situ hybridization, confirming that middle hepatocytes play a key role in the expression of bile acid synthesis genes, and revealing the gene expression profile of hepatocytes in adult mouse liver lobules by zonation and spatial division. This study provides information for the study of liver cell zonation in the human liver. Subsequently, Aizarani et al. (64) performed pseudo- spatial trajectory analysis using scRNA-seq to sequence the arrangement of human hepatocytes in the hepatic lobules, and the results showed similar genetic banding patterns to those observed in mice. Hepatocyte localization is critical to the response of hepatocytes to injury, so further studies on the relationship between hepatocyte zonation and hepatocyte heterogeneity in liver pathology are needed to promote the understanding of the pathogenesis of liver disease.

\section{Liver development and regeneration}

Camp et al. (69) used scRNA-seq to explore the effect of a three-dimensional (3D) culture system on cell characteristics and differentiation, and found that vascular endothelial growth factor (VEGF) was derived from mesenchymal and hepatic lineages within the human liver bud. VEGF enhances endothelial cell proliferation network formation and hepatoblast differentiation, revealing interlineage communication in liver development. Su et al. (70) identified cells with liver and mesenchymal markers coexpressed by Dlk1 and Vimentin, which indicated that epithelial-mesenchymal transition (EMT) or mesenchymalepithelial transition (MET) may be involved in the differentiation of fetal liver stem/progenitor cells (LSPCs) into hepatocytes, Notch signaling in liver development has also been found to be associated with bile duct cell differentiation, further promoting the understanding of the mechanisms of hepatocyte development and LSPC differentiation.

The liver has significant regeneration and repair ability (71). In mouse models of liver injury, the proliferation subsets of hepatocytes were randomly distributed in the periportal 
area, pericentral area, and hepatic lobule (72-74). Aizarani et al. (64) detected some alpha-fetoprotein (AFP) + positive human hepatocytes. MacParland et al. (63) also found the heterogeneity of human hepatocytes and distinguished $\mathrm{AFP}+$ and AFP- hepatocytes, and observed that AFP+ hepatocytes were enriched in cell division and the hepatocyte mitogen signaling pathway interleukin-6 (IL-6). Both $\mathrm{AFP}+$ and $\mathrm{AFP}-$ hepatocytes were distributed in the hepatic lobule, suggesting that $\mathrm{AFP}+$ and $\mathrm{AFP}$ - hepatocytes may possess regenerative ability in liver. Another scRNA-seq data based on human health and regenerative liver epithelia also found a physiological bile acid-induced yes-associated protein (YAP) transcription module, revealing that YAP drives the dynamic heterogeneity of biliary epithelial cells (BECs) and is critical to the response to regenerative duct injury in hepatocytes (75). These studies provide favorable evidence for the heterogeneity and molecular mechanisms of liver cells during liver development, differentiation, and damage repair, and will promote future research on liver development and disease.

\section{Liver immunity and inflammation}

Hepatic immune cells have been shown to regulate key pathological processes in liver diseases, including fibrosis and cancerization (76). In non-alcoholic steatohepatitis (NASH), persistent liver injury triggers recruitment and reprogramming of various immune cells (77). Xiong et al. conducted scRNA-seq on NPCs isolated from the liver of healthy and diet-induced NASH mice and found that NASH-associated macrophages (NAM) were characterized by high expression of the Trem2 receptor, which was related to the severity of the disease and strongly responded to pharmacological and dietary interventions (20). MacParland et al. (63) revealed the presence of two CD68 macrophage populations that are associated with hepatic inflammation and immunoregulatory phenotypes. Other scRNA-seq studies have also defined CD163+MARCO+CD5L+TIMD4+ macrophage populations in human $\mathrm{KCs}$, revealing subpopulations of TREM $2+\mathrm{CD} 9+\mathrm{MNDA}+$ scarassociated macrophages (SAMacs) located in the scar region of fibrotic human liver, which can promote the production and proliferation of HSCs collagen $(19,63,64)$. The ligand receptor interaction model with endothelial cells and PDGFR collagen-producing cells revealed the intrascar activity of TNFRSF12A, PDGFR, and NOTCH fibrophilic pathways (19). In addition, CD8+T enriched population cells and cytotoxic $\mathrm{T}$ lymphocyte-associated 4 $\left(\right.$ CTLA $4^{\text {high }}$ ) regulated $\mathrm{T}$ cells were also identified in human hepatocellular carcinoma (HCC) T cell scRNA-seq, which were associated with impaired antitumor immunity (78). Specific macrophage subsets play a dominant role in the development of chronic liver disease (79). The characteristics of these macrophage subsets in humans will create an important foundation for the study of the role of macrophage subsets in liver disease.

Liver fibrosis is the result of chronic hepatitis, as well as the basic event for the development of cirrhosis or HCC (80). The main characteristic of chronic hepatitis leading to liver fibrosis is the activation of HSCs and their transformation into myofibroblasts (MFB) (81). ScRNAseq study confirmed and expanded the transcriptional characteristics and spatial division of HSCs. During chronic liver disease, HSCs not only produce collagen, but also secrete cytokines. These secreted cytokines are increased in NASH mice, mainly acting on endothelial cells and immune cells, highlighting the important role of HSCs in coordinating the response to liver injury $(17,20,68)$. Dobie et al. (68) also identified that CaHSCs were the main pathogenic collagenic cells in the mouse model of fibrosis, and in the genetic characteristics of CaHSCs, blocking lysophosphatidic acid receptor 1 (LPAR1) could inhibit liver fibrosis in the rodent NASH model. Therefore, LPAR1 may be a potential therapeutic target for liver fibrosis. Krenkel et al. (17) confirmed through fibrotic mouse liver that MFBs are marked by the expression of activated tumor-related protein S100 calcium binding protein A6 (S100a6) and can be further divided into subgroups with immunomodulatory or portal vena fibroblast-like characteristics, showing heterogeneity and functional diversity. In conclusion, scRNA-seq research on liver inflammation has deepened the understanding of the precise cellular and molecular mechanisms of liver fibrosis, and provided favorable support for the rational design and development of highly targeted anti-fiber therapies for patients with chronic liver disease.

\section{Liver tumors and the tumor microenvironment}

The liver is a common site of primary and metastatic tumors. Genetic or genomic variation can lead to different genetic and phenotypic characteristics of cells in tumor tissues, resulting in high heterogeneity of tumor tissues (82). Such high heterogeneity may be related to tumor genesis and metastasis $(83,84)$, and is currently a major obstacle to treatment (85). ScRNA-seq has revealed the single-cell 
transcriptome characteristics of HCC and identified two HCC cell populations characterized by EPCAM differential expression and CD24+/CD44+. CD24+/CD44+-enriched cells specifically express Krt20, S100a6, Vim, and Ctse, among which S100a6 may promote the development of HCC (86). Ma et al. (87) found heterogeneity in HCC cells and the tumor microenvironment (TME), and the degree of heterogeneity was negatively correlated with the prognosis of patients, revealing that the expression of VEGF was correlated with tumor diversity and $\mathrm{T}$ cell dysfunction. This provides a theoretical basis for the combination therapy of immunosuppressive agents and anti-VEGF. Zheng et al. (78) performed scRNA-seq on single T cells from HCC patients, and found that $C c r 8$ and Layn are marker genes for HCC-related regulatory $\mathrm{T}$ cells, suggesting that Layn has an inhibitory function on $\mathrm{T}$ cells and may be a negative regulator involved in tumor killing, and thus, could be a potential therapeutic target for HCC.

The in-depth study of scRNA-seq on liver tumors and the tumor microenvironment is helpful to understand liver tumors and the tumor microenvironment, as well as to discover effective biomarkers, new tumor immunotherapies, and drug therapy targets, which is of great significance for the diagnosis and personalized treatment of liver tumors. Table 2 summarizes the application of scRNA-seq in the liver.

\section{Current challenges and future prospects}

In recent years, transcriptomics has made great strides from the initial study of cell populations to the analysis of

Table 2 Brief overview of scRNA-seq in the liver

\begin{tabular}{|c|c|c|c|c|}
\hline Species & Year & Model & Finding & Reference \\
\hline Human & 2019 & $\begin{array}{l}\text { Healthy development and } \\
\text { liver cirrhosis }\end{array}$ & $\begin{array}{l}\text { Identify a scar-associated subpopulation of } \\
\text { macrophages, define two endothelial cells that } \\
\text { expand in cirrhosis, reveal intra-scar activity of } \\
\text { several pro-fibrogenic pathways }\end{array}$ & Ramachandran et al. Nature (19) \\
\hline Human & 2020 & Healthy development & $\begin{array}{l}\text { Present comprehensively the landscape of liver- } \\
\text { resident immune cells }\end{array}$ & Zhao et al. Cell Discov (21) \\
\hline Human & 2019 & Healthy development & $\begin{array}{l}\text { Construct a human liver cell atlas, reveal } \\
\text { previously unknown subtypes of endothelial cells, } \\
\text { KCs, and hepatocytes }\end{array}$ & Aizarani et al. Nature (64) \\
\hline Mouse & 2017 & Healthy development & $\begin{array}{l}\text { Find that around } 50 \% \text { of liver genes are } \\
\text { significantly zonated and uncover abundant non- } \\
\text { monotonic profiles that peak at the mid-lobule } \\
\text { layers }\end{array}$ & Halpern et al. Nature (67) \\
\hline Human & 2017 & Healthy development & $\begin{array}{l}\text { Reveal interlineage communication regulating } \\
\text { organoid development and Vegf crosstalk } \\
\text { potentiates endothelial Network formation and } \\
\text { hepatoblast differentiation }\end{array}$ & Camp et al. Nature (69) \\
\hline Mouse & 2017 & Healthy development & Identify LSPCs in developing mouse livers & Su et al. BMC Genomics (70) \\
\hline
\end{tabular}

Table 2 (continued) 
Table 2 (continued)

\begin{tabular}{lllll}
\hline Species & Year & Model & Finding & Reference \\
\hline Human & 2017 & Hepatocellular carcinoma & $\begin{array}{l}\text { Identify 11 T cell subsets and delineate their } \\
\text { developmental trajectory }\end{array}$ & Zheng et al. Cell (78) \\
Mouse & 2019 & Liver fibrosis & $\begin{array}{l}\text { Demonstrate the heterogeneity of activated MFB } \\
\text { in vivo and highlight the differences of in vivo and } \\
\text { in vitro activated MFB }\end{array}$ & Krenkel et al. Cells (17) \\
Human & 2019 & Hepatocellular carcinoma & $\begin{array}{l}\text { Reveal two distinct major cell populations } \\
\text { according to EPCAM expression, identify a cell } \\
\text { subpopulation within the EPCAM+ cells }\end{array}$ & Ho et al. Cancer Lett (86) \\
Human & 2019 & Hepatocellular carcinoma & $\begin{array}{l}\text { Find varying degrees of heterogeneity in } \\
\text { malignant cells within and between tumors and } \\
\text { diverse landscapes of tumor microenvironment }\end{array}$ & Ma et al. Cancer Cell (87)
\end{tabular}

scRNA, single cell RNA sequence; KCs, Kupffer cells; HSCs, hepatic stellate cells; LPAR1, lysophosphatidic acid receptor 1; CaHSCs, vena-associated hepatic stellate cells; VEGF, vascular endothelial growth factor; LSPCs, liver stem/progenitor cells, NASH, non-alcoholic steatohepatitis; Trem2, triggering receptor expressed on myeloid cells 2; MFB, myofibroblasts; EPCAM, epithelial cell adhesion molecule.

individual cells. ScRNA-seq has played an important role in identifying new cell subsets, elucidating the trajectory of differentiation, and describing intercellular interactions in a variety of developmental, regenerative, and disease settings. An important aspect of tissue cell heterogeneity is the spatial environment of cells (88). The preparation of liver single-cell suspension leads to the loss of spatial information of liver cells. Meanwhile, single-cell sequencing technology also has problems, such as cumbersome operation, RNA loss in the sequencing process, high detection cost and complex data processing.

In order to ensure that scRNA-seq provides a better service for liver clinical research, we can supplement transcriptome analysis with other "omics" methods to address the above limitations, such as integrating DNA methylation, RNA, protein, and epigenetic modification information to further understand the association between cell phenotypes and genotypes (89). The latest spatial transcriptome technology can trace the gene expression information of cells to the original spatial location in tissues (90). In addition, mass cytometry can conduct an overall process of analysis and imaging mass cytometry (IMC) can acquire spatial information (91). Nowadays, most scRNA-seq studies currently tend to detect freshly isolated cells, we expect that they will tend to explore cryopreservation and immobilization of tissue samples in the future, which will open up clinical research of this technique from a specialized research area to a routine research tool for basic scientists and clinicians. For example, clinical samples can be routinely evaluated on a regular basis, which will provide clinical data to inform the best options for diagnosis, disease progression and treatment. A further experimental improvement in the field of scRNAseq is to increase the sensitivity and accuracy of the assay, thereby reducing the volume of reactions to reduce costs. Alongside technological improvements, computational methods have to be developed in the future, researchers will be able to process and visualise data in a web interface conveniently and quickly. As these technologies develop further, they will reveal multi-dimensional details of the liver microenvironment in physiological and disease states. This will help to promote a new era of precision medicine in hepatology by decoding the molecular mechanisms that regulate human liver disease at single-cell resolution.

With the continuous improvement of scRNA-seq technology, the reduction of sequencing costs, and the progress of precision medicine, scRNA-seq is expected to unlock new avenues for liver biology exploration, liver disease diagnosis, and personalized treatment.

\section{Acknowledgments}

Funding: This work was supported by the Department of Science and Technology of Guizhou Province [Nos. QKHPTRC (2019)5657, QKHZC (2019)2961, QKHZDZXZ (2019)3001, QKHZC (2019)2953, QKHZC (2019)2829, QKHZC (2020)4Y072, QKHZC (2021) normal 420, QKHZC (2021) normal 476, QKHPTRC 
(2017)5733-060 and (2018)5772-001], the Ministry of Science and Technology of China (2017YFC1702005), the Program for Excellent Young Talents of Zunyi Medical University (15zy-004), Department of Education of Guizhou Province [QJHKY (2021)049], and Zunyi City of China [ZSKHSZ (2019)02].

\section{Footnote}

Reporting Checklist: The authors have completed the Narrative Review reporting checklist. Available at https:// dx.doi.org/10.21037/atm-21-4824

Conflicts of Interest: All authors have completed the ICMJE uniform disclosure form (available at https://dx.doi. org/10.21037/atm-21-4824). Dr. AL reports that she serves on Shanghai Nature-Standard Technology Service Co., Ltd., Dr. HL serves on an advisory board for Alnylam pharmaceuticals. The other authors have no conflicts of interest to declare.

Ethical Statement: The authors are accountable for all aspects of the work in ensuring that questions related to the accuracy or integrity of any part of the work are appropriately investigated and resolved.

Open Access Statement: This is an Open Access article distributed in accordance with the Creative Commons Attribution-NonCommercial-NoDerivs 4.0 International License (CC BY-NC-ND 4.0), which permits the noncommercial replication and distribution of the article with the strict proviso that no changes or edits are made and the original work is properly cited (including links to both the formal publication through the relevant DOI and the license). See: https://creativecommons.org/licenses/by-nc-nd/4.0/.

\section{References}

1. Xiong X, Kuang H, Liu T, et al. A Single-Cell Perspective of the Mammalian Liver in Health and Disease. Hepatology 2020;71:1467-73.

2. Si-Tayeb K, Lemaigre FP, Duncan SA. Organogenesis and development of the liver. Dev Cell 2010;18:175-89.

3. Gradilone SA, Masyuk AI, Splinter PL, et al. Cholangiocyte cilia express TRPV4 and detect changes in luminal tonicity inducing bicarbonate secretion. Proc Natl Acad Sci U S A 2007;104:19138-43.

4. Malarkey DE, Johnson K, Ryan L, et al. New insights into functional aspects of liver morphology. Toxicol Pathol 2005;33:27-34.

5. Younossi ZM, Stepanova M, Younossi Y, et al. Epidemiology of chronic liver diseases in the USA in the past three decades. Gut 2020;69:564-8.

6. Udompap P, Kim D, Kim WR. Current and Future Burden of Chronic Nonmalignant Liver Disease. Clin Gastroenterol Hepatol 2015;13:2031-41.

7. Marrone G, Shah VH, Gracia-Sancho J. Sinusoidal communication in liver fibrosis and regeneration. J Hepatol 2016;65:608-17.

8. Wallace MC, Friedman SL, Mann DA. Emerging and disease-specific mechanisms of hepatic stellate cell activation. Semin Liver Dis 2015;35:107-18.

9. Sato K, Marzioni M, Meng F, et al. Ductular Reaction in Liver Diseases: Pathological Mechanisms and Translational Significances. Hepatology 2019;69:420-30.

10. Sato K, Kennedy L, Liangpunsakul S, et al. Intercellular Communication between Hepatic Cells in Liver Diseases. Int J Mol Sci 2019;20:2180.

11. Altschuler SJ, Wu LF. Cellular heterogeneity: do differences make a difference? Cell 2010;141:559-63.

12. Yasen A, Aini A, Wang H, et al. Progress and applications of single-cell sequencing techniques. Infect Genet Evol 2020;80:104198.

13. Fischer DS, Fiedler AK, Kernfeld EM, et al. Inferring population dynamics from single-cell RNA-sequencing time series data. Nat Biotechnol 2019;37:461-8.

14. Tian L, Dong X, Freytag S, et al. Benchmarking single cell RNA-sequencing analysis pipelines using mixture control experiments. Nat Methods 2019;16:479-87.

15. Lotto J, Drissler S, Cullum R, et al. Single-Cell Transcriptomics Reveals Early Emergence of Liver Parenchymal and Non-parenchymal Cell Lineages. Cell 2020;183:702-716.e14.

16. Su T, Yang Y, Lai S, et al. Single-Cell Transcriptomics Reveals Zone-Specific Alterations of Liver Sinusoidal Endothelial Cells in Cirrhosis. Cell Mol Gastroenterol Hepatol 2021;11:1139-61.

17. Krenkel O, Hundertmark J, Ritz TP, et al. Single Cell RNA Sequencing Identifies Subsets of Hepatic Stellate Cells and Myofibroblasts in Liver Fibrosis. Cells 2019;8:503.

18. Yang W, He H, Wang T, et al. Single-Cell Transcriptomic Analysis Reveals a Hepatic Stellate Cell-Activation Roadmap and Myofibroblast Origin During Liver Fibrosis in Mice. Hepatology 2021;74:2774-90.

19. Ramachandran P, Dobie R, Wilson-Kanamori JR, et al. 
Resolving the fibrotic niche of human liver cirrhosis at single-cell level. Nature 2019;575:512-8.

20. Xiong X, Kuang H, Ansari S, et al. Landscape of Intercellular Crosstalk in Healthy and NASH Liver Revealed by Single-Cell Secretome Gene Analysis. Mol Cell 2019;75:644-660.e5.

21. Zhao J, Zhang S, Liu Y, et al. Single-cell RNA sequencing reveals the heterogeneity of liver-resident immune cells in human. Cell Discov 2020;6:22.

22. Kolodziejczyk AA, Kim JK, Svensson V, et al. The technology and biology of single-cell RNA sequencing. Mol Cell 2015;58:610-20.

23. Svensson V, Vento-Tormo R, Teichmann SA. Exponential scaling of single-cell RNA-seq in the past decade. Nat Protoc 2018;13:599-604.

24. Tang F, Barbacioru C, Wang Y, et al. mRNA-Seq wholetranscriptome analysis of a single cell. Nat Methods 2009;6:377-82.

25. Zheng GX, Terry JM, Belgrader P, et al. Massively parallel digital transcriptional profiling of single cells. Nat Commun 2017;8:14049.

26. Goodwin S, McPherson JD, McCombie WR. Coming of age: ten years of next-generation sequencing technologies. Nat Rev Genet 2016;17:333-51.

27. Gross A, Schoendube J, Zimmermann S, et al. Technologies for Single-Cell Isolation. Int J Mol Sci 2015;16:16897-919.

28. Macosko EZ, Basu A, Satija R, et al. Highly Parallel Genome-wide Expression Profiling of Individual Cells Using Nanoliter Droplets. Cell 2015;161:1202-14.

29. Klein AM, Mazutis L, Akartuna I, et al. Droplet barcoding for single-cell transcriptomics applied to embryonic stem cells. Cell 2015;161:1187-201.

30. Gierahn TM, Wadsworth MH 2nd, Hughes TK, et al. Seq-Well: portable, low-cost RNA sequencing of single cells at high throughput. Nat Methods 2017;14:395-8.

31. Islam S, Kjällquist U, Moliner A, et al. Characterization of the single-cell transcriptional landscape by highly multiplex RNA-seq. Genome Res 2011;21:1160-7.

32. Ramsköld D, Luo S, Wang YC, et al. Full-length mRNASeq from single-cell levels of RNA and individual circulating tumor cells. Nat Biotechnol 2012;30:777-82.

33. Picelli S, Faridani OR, Björklund AK, et al. Full-length RNA-seq from single cells using Smart-seq2. Nat Protoc 2014;9:171-81.

34. Jaitin DA, Kenigsberg E, Keren-Shaul H, et al. Massively parallel single-cell RNA-seq for markerfree decomposition of tissues into cell types. Science
2014;343:776-9.

35. Ziegenhain C, Vieth B, Parekh S, et al. Comparative Analysis of Single-Cell RNA Sequencing Methods. Mol Cell 2017;65:631-643.e4.

36. Hedlund E, Deng Q. Single-cell RNA sequencing: Technical advancements and biological applications. Mol Aspects Med 2018;59:36-46.

37. Fan X, Zhang X, Wu X, et al. Single-cell RNA-seq transcriptome analysis of linear and circular RNAs in mouse preimplantation embryos. Genome Biol 2015;16:148.

38. Sheng K, Cao W, Niu Y, et al. Effective detection of variation in single-cell transcriptomes using MATQ-seq. Nat Methods 2017;14:267-70.

39. Gladka MM, Molenaar B, de Ruiter H, et al. Single-Cell Sequencing of the Healthy and Diseased Heart Reveals Cytoskeleton-Associated Protein 4 as a New Modulator of Fibroblasts Activation. Circulation 2018;138:166-80.

40. Svensson V, Natarajan KN, Ly LH, et al. Power analysis of single-cell RNA-sequencing experiments. Nat Methods 2017;14:381-7.

41. Ding J, Adiconis X, Simmons SK, et al. Systematic comparison of single-cell and single-nucleus RNAsequencing methods. Nat Biotechnol 2020;38:737-46.

42. Islam S, Zeisel A, Joost S, et al. Quantitative single-cell RNA-seq with unique molecular identifiers. Nat Methods 2014;11:163-6.

43. Kulkarni A, Anderson AG, Merullo DP, et al. Beyond bulk: a review of single cell transcriptomics methodologies and applications. Curr Opin Biotechnol 2019;58:129-36.

44. Okonechnikov K, Conesa A, García-Alcalde F. Qualimap 2: advanced multi-sample quality control for high-throughput sequencing data. Bioinformatics 2016;32:292-4

45. Wang L, Wang S, Li W. RSeQC: quality control of RNAseq experiments. Bioinformatics 2012;28:2184-5.

46. Hartley SW, Mullikin JC. QoRTs: a comprehensive toolset for quality control and data processing of RNA-Seq experiments. BMC Bioinformatics 2015;16:224.

47. Anders S, Pyl PT, Huber W. HTSeq--a Python framework to work with high-throughput sequencing data. Bioinformatics 2015;31:166-9.

48. Zhang J, Kuo CC, Chen L. WemIQ: an accurate and robust isoform quantification method for RNA-seq data. Bioinformatics 2015;31:878-85.

49. Dobin A, Davis CA, Schlesinger F, et al. STAR: ultrafast universal RNA-seq aligner. Bioinformatics 2013;29:15-21.

50. Liao Y, Smyth GK, Shi W. featureCounts: an efficient 
general purpose program for assigning sequence reads to genomic features. Bioinformatics 2014;30:923-30.

51. Potter SS. Single-cell RNA sequencing for the study of development, physiology and disease. Nat Rev Nephrol 2018;14:479-92.

52. Vallejos CA, Risso D, Scialdone A, et al. Normalizing single-cell RNA sequencing data: challenges and opportunities. Nat Methods 2017;14:565-71.

53. Butler A, Hoffman P, Smibert P, et al. Integrating singlecell transcriptomic data across different conditions, technologies, and species. Nat Biotechnol 2018;36:411-20.

54. Satija R, Farrell JA, Gennert D, et al. Spatial reconstruction of single-cell gene expression data. Nat Biotechnol 2015;33:495-502.

55. Trapnell C, Cacchiarelli D, Grimsby J, et al. The dynamics and regulators of cell fate decisions are revealed by pseudotemporal ordering of single cells. Nat Biotechnol 2014;32:381-6.

56. La Manno G, Soldatov R, Zeisel A, et al. RNA velocity of single cells. Nature 2018;560:494-8.

57. Grün D, Muraro MJ, Boisset JC, et al. De Novo Prediction of Stem Cell Identity using Single-Cell Transcriptome Data. Cell Stem Cell 2016;19:266-77.

58. Matsumoto H, Kiryu H, Furusawa C, et al. SCODE: an efficient regulatory network inference algorithm from single-cell RNA-Seq during differentiation. Bioinformatics 2017;33:2314-21.

59. Van de Sande B, Flerin C, Davie K, et al. A scalable SCENIC workflow for single-cell gene regulatory network analysis. Nat Protoc 2020;15:2247-76.

60. Efremova M, Vento-Tormo M, Teichmann SA, et al. CellPhoneDB: inferring cell-cell communication from combined expression of multi-subunit ligand-receptor complexes. Nat Protoc 2020;15:1484-506.

61. Browaeys R, Saelens W, Saeys Y. NicheNet: modeling intercellular communication by linking ligands to target genes. Nat Methods 2020;17:159-62.

62. Aran D, Looney AP, Liu L, et al. Reference-based analysis of lung single-cell sequencing reveals a transitional profibrotic macrophage. Nat Immunol 2019;20:163-72.

63. MacParland SA, Liu JC, Ma XZ, et al. Single cell RNA sequencing of human liver reveals distinct intrahepatic macrophage populations. Nat Commun 2018;9:4383.

64. Aizarani N, Saviano A, Sagar, et al. A human liver cell atlas reveals heterogeneity and epithelial progenitors. Nature 2019;572:199-204.

65. Ben-Moshe S, Itzkovitz S. Spatial heterogeneity in the mammalian liver. Nat Rev Gastroenterol Hepatol
2019;16:395-410.

66. Han X, Wang R, Zhou Y, et al. Mapping the Mouse Cell Atlas by Microwell-Seq. Cell 2018;172:1091-1107.e17.

67. Halpern KB, Shenhav R, Matcovitch-Natan O, et al. Single-cell spatial reconstruction reveals global division of labour in the mammalian liver. Nature 2017;542:352-6.

68. Dobie R, Wilson-Kanamori JR, Henderson BEP, et al. Single-Cell Transcriptomics Uncovers Zonation of Function in the Mesenchyme during Liver Fibrosis. Cell Rep 2019;29:1832-1847.e8.

69. Camp JG, Sekine K, Gerber T, et al. Multilineage communication regulates human liver bud development from pluripotency. Nature 2017;546:533-8.

70. Su X, Shi Y, Zou X, et al. Single-cell RNA-Seq analysis reveals dynamic trajectories during mouse liver development. BMC Genomics 2017;18:946.

71. Stamataki $Z$, Swadling L. The liver as an immunological barrier redefined by single-cell analysis. Immunology 2020;160:157-70.

72. Lin S, Nascimento EM, Gajera CR, et al. Distributed hepatocytes expressing telomerase repopulate the liver in homeostasis and injury. Nature 2018;556:244-8.

73. Wang B, Zhao L, Fish M, et al. Self-renewing diploid Axin2(+) cells fuel homeostatic renewal of the liver. Nature 2015;524:180-5.

74. Font-Burgada J, Shalapour S, Ramaswamy S, et al. Hybrid Periportal Hepatocytes Regenerate the Injured Liver without Giving Rise to Cancer. Cell 2015;162:766-79.

75. Pepe-Mooney BJ, Dill MT, Alemany A, et al. SingleCell Analysis of the Liver Epithelium Reveals Dynamic Heterogeneity and an Essential Role for YAP in Homeostasis and Regeneration. Cell Stem Cell 2019;25:23-38.e8.

76. Pellicoro A, Ramachandran P, Iredale JP, et al. Liver fibrosis and repair: immune regulation of wound healing in a solid organ. Nat Rev Immunol 2014;14:181-94.

77. Krenkel O, Tacke F. Liver macrophages in tissue homeostasis and disease. Nat Rev Immunol 2017;17:306-21.

78. Zheng C, Zheng L, Yoo JK, et al. Landscape of Infiltrating $\mathrm{T}$ Cells in Liver Cancer Revealed by Single-Cell Sequencing. Cell 2017;169:1342-1356.e16.

79. Bilzer M, Roggel F, Gerbes AL. Role of Kupffer cells in host defense and liver disease. Liver Int 2006;26:1175-86.

80. Dulai PS, Singh S, Patel J, et al. Increased risk of mortality by fibrosis stage in nonalcoholic fatty liver disease: Systematic review and meta-analysis. Hepatology 2017;65:1557-65. 
81. Alegre F, Pelegrin P, Feldstein AE. Inflammasomes in Liver Fibrosis. Semin Liver Dis 2017;37:119-27.

82. Xu Y, Zhou X. Applications of Single-Cell Sequencing for Multiomics. Methods Mol Biol 2018;1754:327-74.

83. Caswell DR, Swanton C. The role of tumour heterogeneity and clonal cooperativity in metastasis, immune evasion and clinical outcome. BMC Med 2017;15:133.

84. Lawson DA, Kessenbrock K, Davis RT, et al. Tumour heterogeneity and metastasis at single-cell resolution. Nat Cell Biol 2018;20:1349-60.

85. Villanueva A. Hepatocellular Carcinoma. N Engl J Med 2019;380:1450-62.

86. Ho DW, Tsui YM, Sze KM, et al. Single-cell transcriptomics reveals the landscape of intra-tumoral heterogeneity and stemness-related subpopulations in liver cancer. Cancer Lett 2019;459:176-85.

Cite this article as: $\mathrm{He} \mathrm{L,} \mathrm{Lu} \mathrm{A,} \mathrm{Qin} \mathrm{L,} \mathrm{Zhang} \mathrm{Q,} \mathrm{Ling} \mathrm{H,}$ Tan D, He Y. Application of single-cell RNA sequencing technology in liver diseases: a narrative review. Ann Transl Med 2021;9(20):1598. doi: 10.21037/atm-21-4824
87. Ma L, Hernandez MO, Zhao Y, et al. Tumor Cell Biodiversity Drives Microenvironmental Reprogramming in Liver Cancer. Cancer Cell 2019;36:418-430.e6.

88. Lee JH. Quantitative approaches for investigating the spatial context of gene expression. Wiley Interdiscip Rev Syst Biol Med 2017;9:e1369.

89. Macaulay IC, Ponting CP, Voet T. Single-Cell Multiomics: Multiple Measurements from Single Cells. Trends Genet 2017;33:155-68.

90. Moncada R, Barkley D, Wagner F, et al. Integrating microarray-based spatial transcriptomics and single-cell RNA-seq reveals tissue architecture in pancreatic ductal adenocarcinomas. Nat Biotechnol 2020;38:333-42.

91. Suvà ML, Tirosh I. Single-Cell RNA Sequencing in Cancer: Lessons Learned and Emerging Challenges. Mol Cell 2019;75:7-12. 